\title{
Effekte einer „optimierten“ Bewegungstherapie auf die Leistungsfähigkeit bei Patienten mit Cystischer Fibrose
}

\author{
U. Rhodius ${ }^{1}$ \\ S. Posselt ${ }^{1}$ \\ H. G. Posselt ${ }^{2}$ \\ R. Hofstetter ${ }^{1}$ \\ D. Leyk ${ }^{3}$
}

\author{
Effects of an Optimized Exercise Therapy on Physical Performance \\ of Patients with Cystic Fibrosis
}

\section{Zusammenfassung}

Körperliches Training soll bei Patienten mit Cystischer Fibrose (CF) zu Leistungssteigerungen und zu einer Besserung der mukoziliären Clearance führen. Ein regelmäßiges Training wird jedoch häufig durch auftretende Infektionen und Zeitmangel eingeschränkt. Das Ziel der vorliegenden Studie war es, die Effekte einer 3-wöchigen Bewegungstherapie unter optimierten Bedingungen auf die Leistungsfähigkeit und Lungenfunktion bei CFPatienten zu untersuchen: 17 Patienten nahmen an einer täglich mindestens 2,5-stündigen Bewegungstherapie unter günstigen klimatischen Bedingungen in einem Sporthotel in Israel (Eilat) teil. Während dieser Zeit erfolgte eine ganztägige Betreuung, die neben vielfältigem Sportangebot eine intensive physikalische Therapie und eine individuelle Ernährungsplanung umfasste. Jeweils 1 Woche vor und nach der Bewegungstherapie sowie nach 7 Monaten wurden die Lungenfunktion überprüft und eine stufenförmige Fahrrad-Spiroergometrie bis zur subjektiven Ermüdung der Probanden durchgeführt. Nach dem 3-wöchigen Training kam es bei der Vitalkapazität und der $\mathrm{FEV}_{1}$ zu 7\%- bzw. $6 \%$-Steigerungen, die allerdings statistisch nicht signifikant waren. Das Training führte jedoch zu signifikanten Verbesserungen der maximalen Leistung (12\%-20\%), der maximalen Sauerstoffaufnahme und des maximalen Atemminutenvolumens. Auch im submaximalen Leistungsbereich traten deutliche Verbesserungen auf (Herzfrequenz, aerob-anaerobe Schwelle). Die Ergebnisse zeigen, dass sich durch eine relativ kurze, aber intensive Be-

\section{Abstract}

Regular exercise training can increase the physical performance of patients with cystic fibrosis (CF). However, training is often hampered by negative factors such as infections, lack of time, etc. The aim of the present study was to investigate the effects of a 3-week-training-program performed under favourable conditions on physical performance and lung function of CF-patients: 17 patients daily trained at least $2,5 \mathrm{~h}$ under suspicious conditions at a sport hotel in Israel (Eilat). During the entire 3 weeks a comprehensive care was applied to the patients including intensive physical therapy and nutrition adapted to the individual demands. Testings of lung function and cycle ergometry ramp tests were performed a week before and after the training program. Additional control measurements were taken 7 months post training. After the 3-week-training vital capacity and FEV1 were increased by $7 \%$ and $6 \%(p>0.05)$. The results of the cycle ergometry showed bigger and significant improvements in the maximal values of power ( $12 \%-20 \%)$, oxygen uptake and ventilation. This findings were also valid for the submaximal exercise range indicated by a slower heart rate slope and a lower aerobicanaerobic threshold. The present results suggest, that relatively large increases in physical performance can be obtained by short, but intensive exercise training including a comprehensive care.

Institutsangaben

${ }^{1}$ Johann Wolfgang Goethe-Universität Frankfurt am Main, Zentrum der Kinderheilkunde und Jugendmedizin, Klinik für Kinderheilkunde II mit dem Schwerpunkt Pädiatrische Kardiologie

${ }_{2}^{2}$ Johann Wolfgang Goethe-Universität Frankfurt am Main, Zentrum der Kinderheilkunde und Jugendmedizin, Klinik für Kinderheilkunde I, Abt. Gastroenterologie

${ }^{3}$ Johann Wolfgang Goethe-Universität Frankfurt am Main, Institut für Sportwissenschaften, Abteilung Sportmedizin

Anmerkung

Grundlage dieses Artikels ist die Dissertationsarbeit von Frau S. Posselt.

Korrespondenzadresse

Dr. U. Rhodius · Klinik für Kinderheilkunde II · Johann Wolfgang Goethe-Universität Frankfurt ·

Theodor-Stern-Kai $7 \cdot 60590$ Frankfurt 
wegungstherapie, die eine umfassende Patientenbetreuung (physikalische Therapie, individuelle Ernährungspläne) einschließt, deutliche Leistungssteigerungen erzielen lassen.

\section{Einleitung}

Die Cystische Fibrose (CF) führt als chronische Erkrankung zu deutlichen Leistungseinschränkungen, die vor allem durch die Störung der Lungenfunktion und durch die Unterernährung infolge Pankreasinsuffizienz entstehen können $[3,8,9]$. In mehreren Studien wurde bereits gezeigt, dass körperliches Training sowohl zu einer positiven Beeinflussung des Krankheitsverlaufes als auch zu einer Steigerung der Leistungsfähigkeit führen kann $[1,7,8,10-13,16]$. Trotz dieser positiven Effekte führt nur ein kleiner Teil der CF-Patienten ein regelmäßiges Training durch: Einerseits wird häufig von erheblichem Zeitmangel berichtet, der u.a. durch die intensive Therapie und durch die Berufstätigkeit verursacht wird. Andererseits besteht aufgrund des körperlichen Handicaps (anhaltende Hustenbeschwerden, Körperbau etc.) für CF-Erkrankte häufig eine Hemmschwelle, mit Gesunden Sport zu treiben. Auch aus Angst vor potenziellen Risiken und ungenügender Betreuung verzichten CF-Patienten auf körperliche Aktivitäten [8]. Ein regelmäßiges Training wird außerdem durch die immer wieder auftretenden Infektionen behindert. Dies kann auch erklären, warum bei Langzeit-Untersuchungen keine deutlichen Trainingseffekte beobachtet wurden [13].

Im Unterschied zu den bisher durchgeführten Untersuchungen absolvierten in der vorliegenden Studie CF-Patienten im Februar 2000 ein 3-wöchiges, allgemeines Fitness-Training unter „optimierten Bedingungen“: Um die Gefahr akuter Atemwegsinfekte zu reduzieren und eine möglichst hohe „Aktivierung“ der Probanden zu erzielen, wurde das Training in einem Sporthotel am Roten Meer (Eilat, Israel) durchgeführt, in dem ganztägig Trainer, Physiotherapeuten, Ernährungswissenschaftler und Ärzte zur Verfügung standen. Im Februar herrschen mit einer Tageshöchsttemperatur von durchschnittlich $21^{\circ} \mathrm{C}$ und einer mittleren Luftfeuchtigkeit von ca. $31 \%$ in Eilat gute Trainingsbedingungen. Da durch das relativ trockene Klima und die vermehrte körperliche Aktivität auch ein veränderter Ernährungsbedarf bestand, wurden individuelle Ernährungspläne erstellt und für zusätzliche Zwischenmahlzeiten sowie entsprechende Enzym- und Flüssigkeitssubstitutionen gesorgt. Ziel war es, den Einfluss einer derart „optimierten“ 3-wöchigen Bewegungstherapie auf die Lungenfunktion und die Ausdauerleistung (Fahrradergometrie) zu untersuchen.

\section{Methode}

An der Studie nahmen 17 CF-Patienten (7 weiblich, 10 männlich) mit den folgenden anthropometrischen Daten teil: Alter 28,5 \pm 9,6 Jahre $(\bar{x} \pm S D)$, Gewicht $55,5 \pm 8,5 \mathrm{~kg}$, Größe $169,5 \pm 6,4 \mathrm{~cm}$, Bodymass-Index 19,3 $\pm 2,6$. Die Probanden wurden jeweils 1 Woche vor und nach der 3-wöchigen Bewegungstherapie in Israel (Eilat) untersucht. 7 Monate nach dem Training wurde eine abschließende Kontrolluntersuchung durchgeführt, an der krankheitsbedingt nur 16 Probanden teilnahmen. Ausschlusskriterien für die Messungen waren die akute Exazerbation eines Atemweginfektes und eine transkutane Sauerstoffsättigung $(<90 \%)$.

Während des 3-wöchigen Aufenthaltes wurden die CF-Patienten von einem Physiotherapeuten (Atemgymnastik, Klopfdrainage etc.), einer Ernährungswissenschaftlerin, mehreren Bewegungstherapeuten und Ärzten ganztägig betreut. Um den erhöhten Ernährungs-/Elektrolytbedarf zu decken, wurde für jeden Probanden eine spezielle, individuelle Enzym- und Flüssigkeitssubstitution durchgeführt und zusätzliche Zwischenmahlzeiten verabreicht. Die Medikation wurde nicht verändert. Vereinbarungsgemäß bestand für die Probanden die Verpflichtung, täglich an einem 2,5-stündigen Trainingsprogramm teilzunehmen. Darüber hinaus wurden den Patienten weitere sportliche Aktivitäten (u.a. Beach-Volleyball, Basketball, Tennis, Bogenschießen, Gymnastik, Schwimmen) angeboten, die von der überwiegenden Anzahl der Studienteilnehmer gerne genutzt wurden. Der Tagesablauf ist in Tab. 1 dargestellt.

Tab. 1 Tagesablauf während des 3-wöchigen Aufenthaltes in Eilat (Israel)

\begin{tabular}{ll}
\hline $08.30-09.00$ & „Pflichtsport“I: Gymnastik, Aufwärm-/Dehnungsübungen, \\
& Walking, Fangspiele etc. \\
\hline $09.00-10.00$ & Frühstück \\
\hline $10.00-10.30$ & Therapie \\
\hline $10.30-12.30$ & „Pflichtsport“ II: Basket-, Volley-, Fuß-, Völker-, Wasserball, \\
& Zirkeltraining, Badminton, Tennis, Tischtennis, Schwimmen \\
\hline $12.30-13.00$ & Pause \\
\hline $13.00-14.00$ & Mittagessen \\
\hline $14.00-15.00$ & Mittagspause/Therapie \\
\hline Ab 15.00 & Freizeitaktivitäten/Club-Angebot \\
\hline $18.00-19.00$ & Fortbildung/Beratung \\
\hline 20.00 & Abendessen \\
\hline
\end{tabular}

Die Vitalkapazität $(\mathrm{VC})$, die Einsekundenkapazität $\left(\mathrm{FEV}_{1}\right)$ und die ventilatorischen Parameter Atemminutenvolumen (VE), Sauerstoffaufnahme $\left(\mathrm{VO}_{2}\right)$, Kohlendioxydabgabe $\left(\mathrm{VCO}_{2}\right)$ wurden mit Hilfe des Sensormedics-Vmax229-Gerätes bestimmt (Fa. Sensormedics, Yorbalinda, Kalifornien, USA). Bei der Überprüfung der Lungenfunktion, die immer vor der Spiroergometrie durchgeführt wurde, atmeten die Patienten durch ein Mundstück mit zwischengeschaltetem Bakterienfilter der Fa. Sensormedics. Während der fahrradergometrischen Belastung trugen die Probanden eine Gesichtsmaske (Fa. Hans Rudolph, Kansas City, USA). Die Atemgase wurden online in einer breath-to-breathAnalyse registriert. Aus den ermittelten $\mathrm{VO}_{2}$-und $\mathrm{VCO}_{2}$-Werten wurden außerdem der respiratorische Quotient und die aerobanaerobe Schwelle berechnet. Die Herzfrequenzen (HF) wurden über ein 12-Kanal-EKG kontinuierlich abgeleitet (Case 19, Fa. Marquette-Hellige, Freiburg, BRD). Außerdem wurde der Blutdruck (Ergoline Ergometrics 900, Binz, BRD) und die transkutane Sauerstoffsättigung (Nellcor Escort MDE, Arleta, Kalifornien, USA) während der Belastung fortlaufend überwacht.

Die Ergometrie wurde in halbliegender Position auf einem Fahrradergometer (Ergoline Ergometrics 900, Binz, BRD) durchgeführt. Nach einer initialen kurzen Aufwärmphase (lastfrei) wurde die Belastungsintensität alle $60 \mathrm{~s}$ um $20 \mathrm{~W}$ bis zum Abbruch 

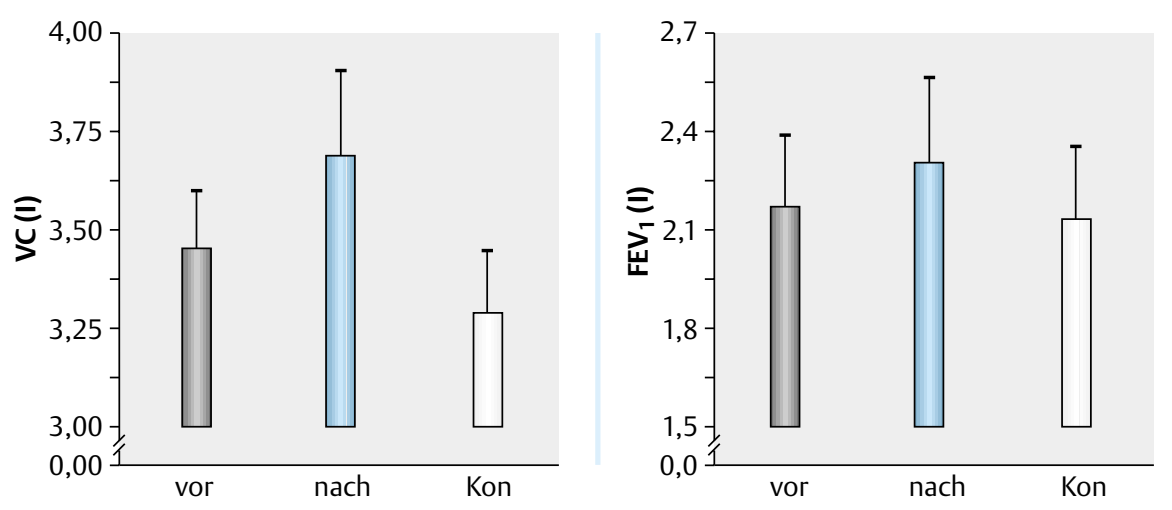

Abb. 1 Vitalkapazität (VC) und Forciertes Expiratorisches Volumen der 1. Sekunde $\left(\mathrm{FEV}_{1}\right)$ vor, nach (jeweils 7 Tage) sowie 7 Monate im Anschluss („Kon“) an eine 3-wöchige Bewegungstherapie am Roten Meer $(\bar{x} \pm S E, n=17)$ aufgrund pulmonaler oder muskulärer Erschöpfung gesteigert (Belastungsprotokoll nach Godfrey 1974). Die Ergometrie wurde beendet, wenn die Probanden nicht mehr in der Lage waren, eine Trittfrequenz von mindestens 60 Umdrehungen/min aufrechtzuhalten oder die als untere Grenze gesetzte transkutane Sauerstoffsättigung von $90 \%$ unterschritten wurde.

Bei der Mittelwertsdarstellung $(\bar{x})$ wurden als Streuungsmaße im Text bzw. Tabellen die Standardabweichung (SD) und in den Abbildungen der Standardfehler (SE) verwendet. Mit Hilfe einer einfaktoriellen Varianzanalyse (Faktor Messwiederholung) wurde das Körpergewicht, die Lungenfunktionsdaten und die Maximalwerte aus der Fahrradergometrie untersucht. Im submaximalen Bereich wurden die Versuchsergebnisse mit Hilfe einer zweifaktoriellen Varianzanalyse (Faktoren Messwiederholung und Intensität) analysiert. Lagen signifikante Einflüsse $(p<0,05)$ vor, wurden multiple Zellvergleiche als post-hoc-Test nach dem Verfahren von Newman-Keuls durchgeführt.

\section{Ergebnisse}

Abb.1 zeigt die Ergebnisse der Lungenfunktionstests. Die höchsten Werte in der Vitalkapazität (VC) (7\% Zunahme) und im Forcierten Exspiratorischen Volumen der 1. Sekunde $\left(\mathrm{FEV}_{1}\right)(6 \% \mathrm{Zu}-$ nahme) werden nach der 3-wöchigen Bewegungstherapie am Roten Meer erreicht. Allerdings sind die Unterschiede zu den Ausgangswerten und zur Kontrolluntersuchung statistisch nicht signifikant $(p>0,05)$. Dies gilt nicht für die fahrradergometrisch bestimmte Maximalleistung: Nach der 3-wöchigen Bewegungstherapie werden mit 2,24 $\pm 0,65 \mathrm{~W} / \mathrm{kg}$ Körpergewicht signifikant höhere Leistungen erreicht, die etwa $12 \%$ bzw. $20 \%$ über den Vergleichswerten liegen $(p<0,05)$ (Abb. 2).

Die maximale Sauerstoffaufnahme, das maximale Atemminutenvolumen und die aerob-anaerobe Schwelle (Abb.3) liegen nach der 3-wöchigen Bewegungstherapie ebenfalls deutlich über den entsprechenden Vergleichswerten $(p<0,05)$, während sich die maximalen Herzfrequenzen nicht signifikant voneinander unterscheiden. In Abb. 4 ist zu sehen, dass im submaximalen Bereich der Herzfrequenzanstieg nach der 3-wöchigen Bewegungstherapie deutlich geringer ausfällt $(p<0,05)$. Die Sauerstoffaufnahmen unterscheiden sich kaum voneinander $(p>0,05)$.

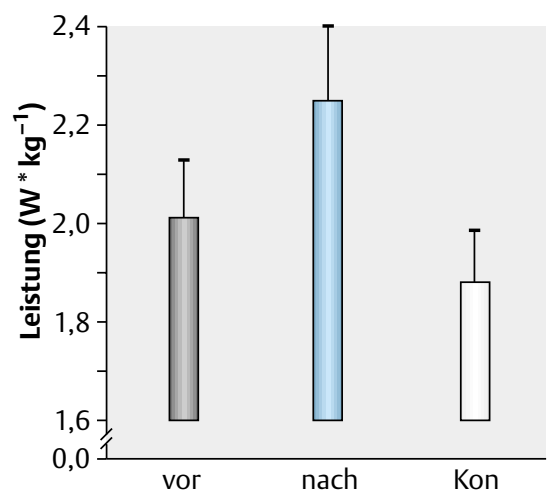

Abb. 2 Maximale Leistung bei erschöpfender Fahrradergometrie vor, nach (jeweils 7 Tage) sowie 7 Monate im Anschluss („Kon“) an eine 3-wöchige Bewegungstherapie am Roten Meer ( $\bar{x} \pm S E, n=17)$.

\section{Diskussion}

In der vorliegenden Studie wurden die Effekte einer Bewegungstherapie unter günstigen klimatischen Bedingungen untersucht, in der nicht nur über ein vielfältiges, ganztägiges Sport- und Therapieangebot alle motorische Hauptbeanspruchungsformen trainiert werden konnten, sondern auch eine umfassende Patientenbetreuung erfolgte (s. Tab.1). Der Erfolg der unterstützenden Maßnahmen kann u. a. auch aus der geringfügigen Zunahme des Körpergewichtes $(0,75 \mathrm{~kg})$ abgeleitet werden, obwohl die Probanden im Vergleich zu ihrem gewohnten Tagesablauf in Eilat körperlich wesentlich aktiver waren. Unsere Ergebnisse zeigen, dass es auch in einer relativ kurzen Zeitspanne (3 Wochen) zu deutlichen Leistungssteigerungen kommen kann.

Diese positiven Effekte haben allerdings keinen wesentliche Einfluss auf die Lungenfunktionswerte; die Verbesserungen betragen zwar bei der Vitalkapazität 7 bzw. $6 \%$ bei der $\mathrm{FEV}_{1}$ (Abb.1), statistisch sind sie aber nicht signifikant. Dieser Befund wird durch zahlreiche frühere Untersuchungen bestätigt $[1,4,5$, $7,12,14]$. Schneiderman-Walker u. Mitarb. [13] haben in ihrer 3-jährigen Längsschnittstudie allerdings festgestellt, dass die Abnahme der Vitalkapazität in der „Trainingsgruppe“ deutlich geringer ausfiel als in dem Kontrollkollektiv. Ein möglicher Effekt auf die Vitalkapazität kann letztlich auch hier nicht ausgeschlossen werden: Das Signifikanz-Niveau wird mit einem p von 0,079 nur knapp verfehlt, dies kann aber auch mit der Anzahl $(\mathrm{n}=17)$ und der Homogenität des untersuchten Kollektives zusammenhängen. Als ein Indiz für eine Verbesserung der Lungenfunktion 

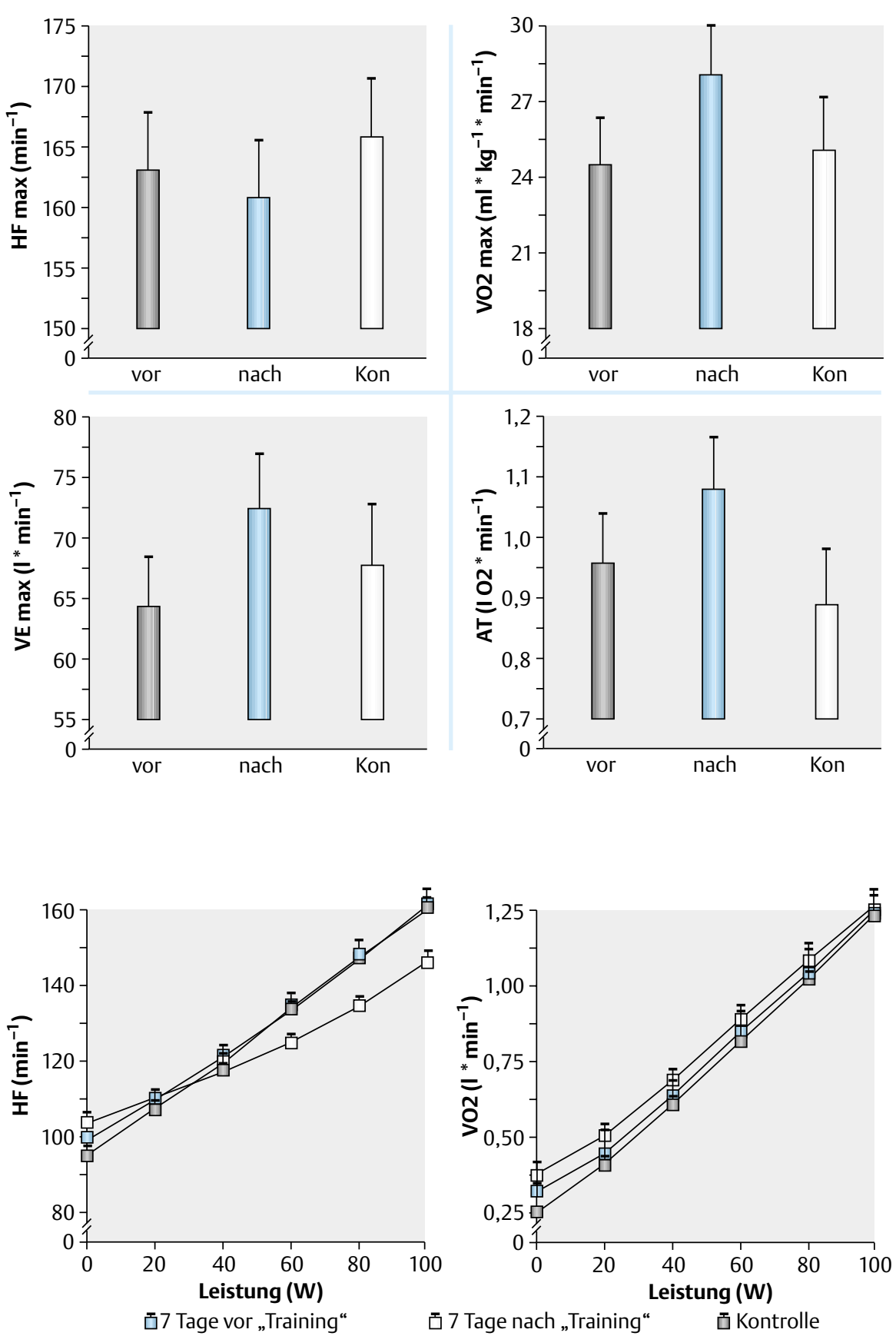

Abb. 3 Maximale Herzfrequenz (HF), Sauerstoffaufnahme $\left(\mathrm{VO}_{2 \max }\right)$, Atemminutenvolumen (VE) und anaerobe Schwelle (AT) während erschöpfender Fahrradergometrie $(\bar{x} \pm S E, n=17)$. Die Tests wurden vor und nach (jeweils 7 Tage) sowie 7 Monate („Kon“) im Anschluss an eine 3-wöchige Bewegungstherapie am Roten Meer durchgeführt.
Abb. 4 Submaximale Herzfrequenzen (HF) und Sauerstoffaufnahmen $\left(\mathrm{VO}_{2}\right)$ während stufenförmiger Fahrradergometrie vor, nach (jeweils 7 Tage) sowie 7 Monate im Anschluss („Kontrolle“) an eine 3-wöchige Bewegungstherapie am Roten Meer $(\bar{x} \pm S E$, $\mathrm{n}=17$ ). Die „0-Watt-Belastung“ ist dem "Leer-Treten" auf dem Fahrradergometer gleichzusetzen. könnten auch die Änderungen der ventilatorischen Parameter während der Fahrradergometrie angesehen werden (Abb. 3).

Nach der 3-wöchigen Trainingstherapie kam es im stufenförmigen Belastungstest zu einer deutlichen Leistungssteigerung mit signifikanten Verbesserungen der leistungsphysiologischen Kenngrößen (s. Abb. 2-4). Diese zeigen sich nicht nur im maximalen sondern auch im submaximalen Leistungsbereich (Herzfrequenz, aerob-anaerobe Schwelle), der im Alltag natürlich eine höhere Relevanz hat. In Abb. 4 ist gut zu erkennen, dass körperliches Training $\mathrm{zu}$ einem geringeren Herzfrequenzanstieg führt. Die einfach zu messende Herzfrequenz eignet sich somit gut für die Trainingskontrollen im submaximalem Bereich. Anhand der bei der Spiroergometrie beim Unterschreiten des Grenzwertes der transkutanen Sauerstoffsättigung (90\%) ermittelten Herzfrequenz können durch Pulskontrolle Belastungen vermieden werden, die zu einer Sauerstoffunterversorgung während des Sports führen.

In den Kontrolluntersuchungen, die 6-8 Monate nach dem Aufenthalt in Israel durchgeführt wurden, wird allenfalls das ursprüngliche Leistungsniveau erreicht. Eine abschließende Befragung ergab, dass die Probanden natürlich nicht unter den vergleichbaren Bedingungen weiter trainieren konnten und somit langfristige Trainingseffekte auch nicht zu erwarten sind [18].

In anderen Studien wurde häufig der Effekt eines mehrmonatigen Trainings untersucht, in dem die Patienten mehrmals pro Woche eine Sportart (z.B. Schwimmen, Radfahren, Laufen) betrieben $[5,7,12,14,15]$. Aufgrund der recht unterschiedlichen Belastungsnormative (Intensität, Umfang) erstaunt es kaum, dass die Angaben über die erzielten Leistungsänderungen divergie- 
ren. Im Vergleich zu einigen anderen Untersuchungen wurden mit der kurzen, aber intensiven und umfassenden Therapie in Eilat sogar deutlich höhere Leistungssteigerungen erzielt $[4,13,17]$. Wir führen die positiven Effekte auf die Kombination aus breitem Sportangebot, intensiver physikalischer Therapie und individuellem Ernährungsplan zurück. Unter diesen günstigen Bedingungen scheint es möglich zu sein, auch in relativer kurzer Zeit Leistungssteigerungen zu erzielen, dies wurde von den beteiligten Patienten als motivierend bewertet.

\section{Literatur}

${ }^{1}$ Andreasson B, Jonson B, Kornfält R et al. Longterm effects of physical exercise on working capacity and pulmonary function in cystic fibrosis. Acta Paediatr Scand 1998; 776: 70-75

${ }^{2}$ Baldwin DR, Hill AL, Peckham DG et al. Effect of addition of exercise to chest physiotherapy on sputum expectoration and lung function in adults with cystic fibrosis. Respir Med 1994; 88: 49-53

3 Behrman RE, Kliegman RM, Jenson HB. The Respiratoy System Chapter 416. In: Nelson, Textbook of pediatrics. Philadelphia: WB Saunders Company, 2000: 1315-1327

${ }^{4}$ Braggion C, Cornacchia M, Miano A et al. Exercise tolerance and effects of training in young patients with cystic fibrosis and mild airway obstruction. Pediatr Pulmonol 1989; 7: 145-152

${ }^{5}$ Edlund LD, French RW, Herbst JJ et al. Effects of a swimming program on children with cystic fibrosis. Am J Dis Child 1986; 140: 80-83

${ }^{6}$ Godfrey S. Exercise testing in children. Applications in health and disease. London, Philadelphia, Toronto: W.B. Saunders Company Ltd, 1974: S $28-101$
${ }^{7}$ Gulmans VAM, DeMeer K, Brackel HJL et al. Outpatient exercise training in children with cystic fibrosis: physiological effects, perceived competence, and acceptability. Pediatr Pulmonol 1999; 28: 39-46

${ }^{8}$ Hebestreit H, Kriemler S, Hebestreit A. Körperliche Aktivität und Training bei Mukovizidose. D Zeitschr Sportm 2000; 51: 85-93

${ }^{9}$ Klinger KW. Genetics of cystic fibrosis. Semin Respir Med 1985; 6: $243-251$

${ }^{10}$ Moorcroft AJ, Dodd ME, Webb AK. Exercise testing and prognosis in adult cystic fibrosis. Thorax 1997; 52: $291-293$

${ }^{11}$ Nixon PA. Role of exercise in the evaluation and management of pulmonary disease in children and youth. Med Sci Sports Exerc 1996; 28 : $414-420$

12 Orenstein DM, Franklin BA, Doershuk CF et al. Exercise conditioning and cardiopulmonary fitness in cystic fibrosis. The effects of a threemonth supervised running program. Chest 1981; 80: 392 - 398

${ }^{13}$ Schneiderman-Walker J, Pollok SL, Corey M et al. A randomized controlled trial of a 3-year home exercise program in cystic fibrosis. J Pediatr 2000; 136: 304-309

${ }^{14}$ Stanghelle JK, Hjeltnes N, Bangstad HJ et al. Effect of daily short bouts of trampoline exercise during 8 weeks on the pulmonary function and the maximal oxygen uptake of children with cystic fibrosis. Int J Sports Med 1988; 9: 32-36

15 Strauss GD, Osher A, Wang CI et al. Variable weight training in cystic fibrosis. Chest 1987; 92: 273-276

${ }^{16}$ Thomas J, Cook DJ, Brooks D. Chest physical therapy management of patients with cystic fibrosis. Am J Respir Crit Care Med 1995; 151: $846-850$

${ }^{17}$ Zach M, Oberwaldner B, Häusler F. Cystic fibrosis: Physical exercise versus chest physiotherapy. Arch Dis Child 1982; 57: 587-589

${ }^{18}$ Zach M, Purrer B, Oberwalder B. Effect of swimming on force expiration and sputum clearance in cystic fibrosis. Lancet 1981; 2 $1201-1203$ 